\title{
Dissociable Temporal Lobe Activations during Emotional Episodic Memory Retrieval
}

\author{
R. J . Dolan,*,† R. Lane, P. Chua,‡ and P. Fletcher* \\ *Wel I come Department of Cognitive Neurology, Institute of Neurology, Queen Square, London, WC1N 3BG; †Royal FreeH ospital School \\ of Medicine, Roland Hill Street, London, NW3, United Kingdom; and ¥Department of Psychiatry, \\ The Royal MelbourneHospital, Grattan Street, Parkville, Victoria 3050, Australia
}

Received August 3, 1999

The richness of human recollective experience is, in part, related to evocation of previously experienced emotions. An extensive functional neuroi maging literature has provided a description of brain regions involved in retrieving emotionally neutral episodic memories. Whether similar or distinct systems are involved in retrieving emotional memories is unresolved. This question motivated the present functional neuroimaging study, using 0-15 positron emission tomography (PET), where we compared patterns of brain activation associated with retrieving previously studied emotional and neutral pictorial material. By varying task requirements and item density we characterized two distinct neural response patterns during emotional memory retrieval. First, we identified an anterior temporal pole activation that reflected the psychological set associated with emotional memory retrieval. Second, we identified a left amygdala response sensitive to actual retrieval of emotional items. These data suggest distinct functional roles for temporal lobe regions during emotional memory retrieval involving context-related tonic anterior temporal pole activation and a phasic item-related amygdala response. We conclude that brain regions involved in episodic memory retrieval reflect not only physical attributes of stimulus material, for example, their verbal or visual qualities, but also their affective significance. 02000 Academic Press

\section{INTRODUCTION}

An important, though frequently overlooked, component of autobiographical memory is an associated emotional tone that, to varying degrees, is a quality of most recollective experience. Indeed, a cl ose functional relationship between memory and emotion is supported by a number of lines of evidence that include enhanced learning for events with strong emotional valence (Bower, 1992) and preferential recall for emotionally laden autobiographical events (Holmes, 1970; Brewer,
1988). In this study we address the question of whether neural systems known to beinvolved in epi sodic memory retrieval also subserve retrieval of emotional episodic memories. The alternative possibility, based on a transfer appropriate processing model, would suggest that emotional memory retrieval involves systems known to be involved in acquisition of emotional memories (McGaugh et al., 1996).

Our experimental approach involved comparing conditions where the valence of pictorial material (emotional versus neutral), task requirement (recognition memory or a judgement task), and density of target items (high versus low) were experimentally manipulated. In essence our design involved three factors (task, valence, and target density), each with two levels (see Fig. 1). As this design involved presentation of old and new items at test (corresponding to high and low target density conditions), it is important to control for nonspecific effects, such as emotional arousal, elicited by on-line processing of targets and foils, particularly during emotional recognition. This issue is addressed by the judgement condition, where valence and target density is varied in an identical manner to the memory conditions.

\section{METHODS}

Ten healthy male vol unteer subjects who were free of past and present neurological and psychiatric disorder were recruited for the study. N one of the subjects were taking current medication. All patients gave written informed consent. The study was approved by the J oint Ethics Committee of the National Hospital for Neurology and Neurosurgery and I nstitute of Neurology.

\section{ST UDY MATERIAL AND PSYCHOLOGICAL TASKS}

The study items were visual images of objects or scenes drawn from the International Affective Pictorial 


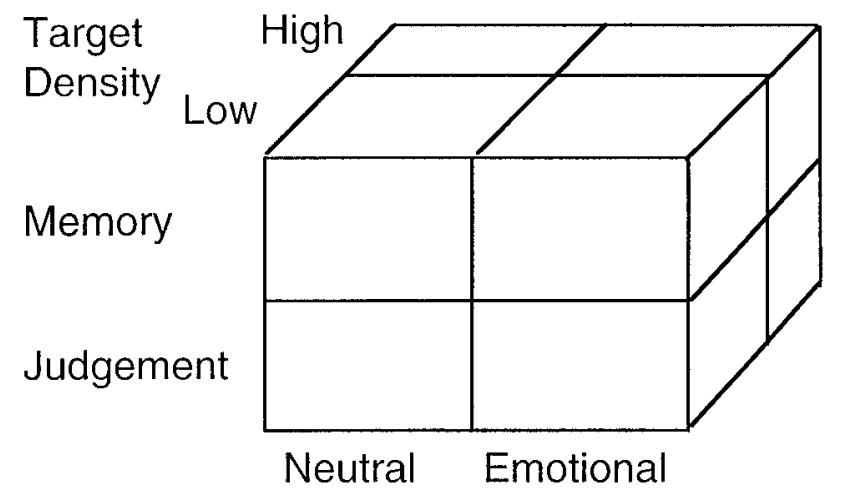

FIG. 1. The experimental design involving three conditions each with two levels. These comprise task (memory or judgement), target density (high versus low), and valence of material (emotional or neutral).

System (IAPS), which consists of images standardized with respect to emotional valence and arousal ( $L$ ang et al., 1995). The items consisted of two discrete valence categories, reflecting either neutral or emotional content. The emotional content pictures consisted either positively or negatively valenced (i.e., pleasant or unpleasant) items. Twelve study sets were constructed containing lists of 20 items and for each of these study lists a corresponding test list was constructed. These test lists varied with respect to the number of study items they contained (High target density $=80 \%$ targets; Low target density $=0 \%$ targets).

Five minutes prior to each scanning window subjects were shown a study set of pictures consisting of either emotional or neutral items. Pictures were displayed for $3 \mathrm{~s}$ each during this encoding phase. The task requirement was for the subjects to make a val ence rating, on a 9-point scale, for each of the pictures. Following each scan (following test) subjects gave one arousal rating for the entire picture set viewed during the scan. Valence ratings range from 1 (extremely unpleasant) to 9 (extremely pleasant). Arousal ratings range from 1 (extremely calm) to 9 (extremely aroused). These ratings were obtained using a rating scale, the SelfAssessment Mannikan (SAM) (Bradley and Lang, 1994). These ratings were highly correlated with valence norms from American subjects supporting the validity of the use of IAPS photos in British subjects. Between study and test, performed during scanning, subjects were engaged in a distracter task involving serial subtraction, for $2 \mathrm{~min}$, to prevent item rehearsal. Experimental conditions were counterbalanced across subjects.

The psychological tasks involved either a visual recognition or judgement task. During scanning subjects were presented with picture sequences, some of which they had previously seen at study. Pictures were displayed for $6 \mathrm{~s}$ followed by a blank screen for $1 \mathrm{~s}$ $(I S I=7 \mathrm{~s})$. As outlined the proportion of study items contained in these test sequences varied from either 85 or $0 \%$, constituting high and low target conditions, respectively. This manipulation of target density was crossed with valence (emotional or neutral conditions) and with task (recognition or judgement conditions).

In the memory recognition task subjects responded with a yes/no response to indicate whether they had seen each individual item at study. In the judgement task subjects made an outdoor/indoor judgement. The proportions of binary responses in this condition was tailored to match that of the memory task, i.e., outdoor responses were similar in number to correct high and low target responses in the memory condition.

Valence and arousal ratings were obtained at the conclusion of each scan, which confirmed that valence ratings for unpleasant $(t=5.05 ; \mathrm{df}=10 ; \mathrm{P}<0.001)$ and pleasant $(t=2.2 ; d f=11 ; P=0.05)$ picture sets were significantly different from neutral picture sets in the predicted direction. These findings indicate that pictures classified as pleasant, unpleasant, and neutral were experienced as such during neuroimaging. Selfreported arousal ratings for pleasant (mean $=4.39$; $\mathrm{SD}=1.69)$, unpleasant (mean $=5.14 ; \mathrm{SD}=2.24)$, and neutral (mean $=3.59$; SD $=2.01$ ) pictures did not differ significantly. The correlations between scanning measures and normative data were 0.97 for valence and 0.79 for arousal ratings. Since arousal ratings for emotional pictures typically exceed those of neutral pictures, these findings suggest that the psychological task requirements attenuated the intensity of the associated emotional arousal.

\section{FUNCTIONAL IMAGING}

Overall 12 perfusion scans were acquired, to index condition-specific neural activity, using a SIEMENS/ CPS ECAT EXACT HR + (model 962) PET scanner (Siemens/CTI, Knoxville, TN) in 3-D mode with a 15-cm axial field of view. Relative rCBF was measured from the distribution of radioactivity after slow bolus i.v. injection of $\mathrm{H}_{2}{ }^{15} \mathrm{O}$ (9 mCi per scan, each lasting $90 \mathrm{~s}$ ). Attenuation-corrected data were reconstructed into 63 image planes with a resulting resolution of $6 \mathrm{~mm}$ at full-width-half-maximum. For each subject, structural magnetic resonance (MR) images were obtained with a 2T Magnetom VISION scanner (Siemens, Erlangen Germany).

\section{STATISTICAL AN ALYSIS}

Statistical parametric mapping (SPM96, Wellcome Department of Cognitive Neurology, London, UK) software was used for image realignment, transformation into standard stereotactic space, smoothing, and statis- 
tical analysis (Friston et al., 1995). All measurements per condition were averaged across subjects. Statedependent differences in global flow were covaried out using ANCOVA. Main effects and interactions were assessed with contrasts of the adjusted task means using t statistics subsequently transformed into the $z$ statistic. Corrected values refer to correction for the whole brain volume based upon magnitude of the response. The resulting set of $z$ values constituted a statistical parametric map (SPM $\{z\})$. Localization of maxima are reported within the standard space as defined by Talairach and Tournoux and were superimposed on the group mean magnetic resonance image spatially normalized in to the same anatomical space (Talairach and Tournoux, 1988).

The main hypothesis being tested in this experiment was whether the amygdala is engaged as a function of emotional memory retrieval. As we were particularly interested in activations associated with intentional emotional retrieval we compared retrieval of emotional versus neutral items in an explicit memory task with emotional versus neutral items in a judgement task. Note that in the latter condition any retrieval is likely-to-incidental. In this comparison high and low target density conditions are combined. To directly examine the influence of target density, involving actual retrieval of emotional items, a subsequent analysis included target density as a factor. For assessing statistical significance of regional activations we use corrected significance levels for activations not predicted a priori and uncorrected levels for activations that were predicted. Predicted activations during retrieval include right prefrontal cortex, parietal cortex, and medial temporal lobes (areas we have found in previous studies).

\section{RESULTS}

Behavioral data at test are summarized in Table 1. These data show that performance was virtually at ceiling with no significant performance differences evident across conditions.

\section{TABLE 1}

Behavioral Performance Data as a Function of Valence, Target Density (High and Low Target Density), and Psychological Task Conditions

\begin{tabular}{lcccccc}
\hline & Emotional & Neutral & High & Low & Mem. & J udg. \\
\hline Hits & 0.98 & 0.95 & 0.97 & 0.97 & 0.98 & 0.96 \\
FalseAlarm & 0.07 & 0.11 & 0.07 & 0.09 & 0.01 & 0.15 \\
d' & 3.52 & 2.87 & 3.36 & 3.22 & 4.38 & 2.78 \\
\hline
\end{tabular}

Note Hits refers to old items correctly identified as old; false alarms refers to new items incorrectly identified as old; and d' is a measure accuracy taking into account response bias.

\section{TABLE 2}

Coordinates and Z Scores for Regions Activated in Distinct Contrasts

\begin{tabular}{lll}
\hline Region & Coordinates $x, y, z$ & Z Score \\
\hline
\end{tabular}

(a) Emotional versus neutral memory conditions

$\begin{array}{lcc}R \text { ant. temporal pole } & 60,8,-18 & 5.0^{*} \\ \mathrm{~L} \text { ant. temporal cortex } & -48,-10,-4 & 3.93\end{array}$

(b) High versus low target in emotional versus neutral memory Left amygdala

$-12,4,-26$

4.18

(c) Recognition memory (high versus low target) related activations

$\mathrm{R}$ anter. prefrontal cortex

$\mathrm{L}$ anter. prefrontal cortex

$26,62,4$

3.86

R. post. parietal cortex

$-30,52,0$

3.73

3.61

$* \mathrm{P}<0.05$ corrected.

The critical analysis in this study is whether emotional content influenced episodic retrieval related activations. We addressed this by comparing neural responses associated with retrieving emotional compared to neutral items (low and high target conditions combined) versus the same comparison in the judgement conditions. This contrast revealed an highly significant activation in right anterior temporal pole $(P<0.05$, corrected) specific to the emotional memory condition (see Fig. 2; Table 2a). An activation in left amygdala was also evident in this contrast at a lower level of significance ( $P<0.001$, uncorrected).

The profile of activation we observed in anterior temporal pole, associated with emotional memory retrieval, is open to two interpretations. First, the finding might reflect an effect of psychological set engendered by engaging in emotional memory retrieval. It follows that this type of response, within the constraints of our study, should be independent of emotional item target density. Alternatively, the pattern might reflect actual retrieval of emotional memories and index a target density retrieval effect. Note that it remains possible that there is incidental retrieval in the judgment condition and this contrast best characterizes effects related to intentional retrieval.

To distinguish between psychological set-related and target density effects, during emotional memory re trieval, we carried out two additional analyses. First, we compared activations associated with emotional versus neutral memory retrieval in the zero target conditions alone to rule out target density effects on right temporal pole activation. The right temporal pole remained significant in this comparison $(P<0.001$, uncorrected) with additional activations seen in bilateral parahippocampal gyri ( $\mathrm{P}<0.001$, uncorrected). Second, we directly assessed the influence of emotional item target density on retrieval-related activations. 


\section{sagittal}

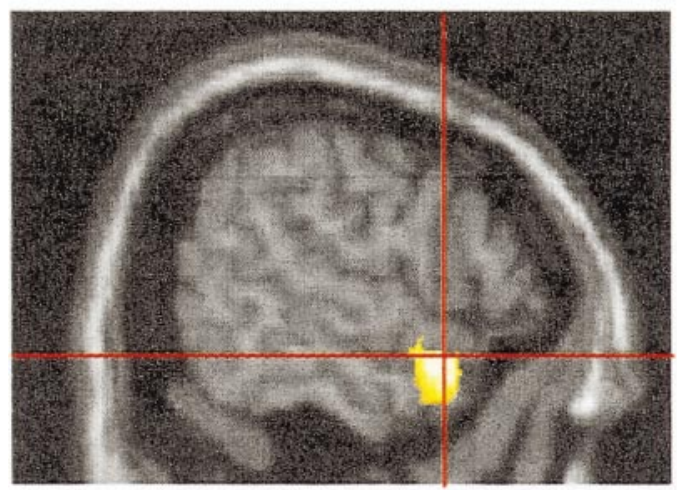

\section{coronal}

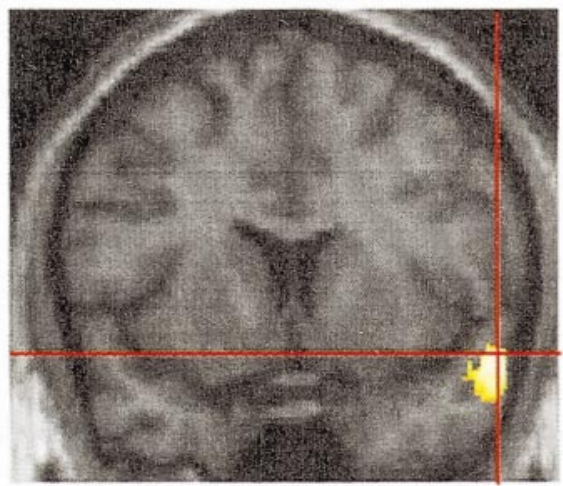

\section{transverse}

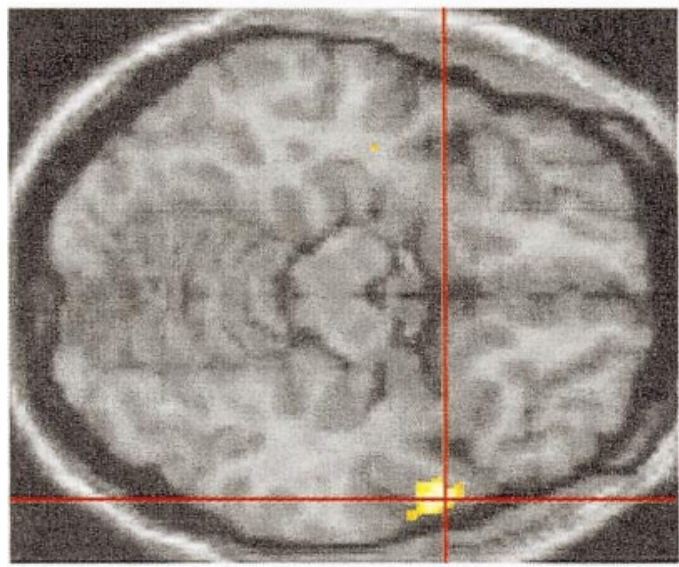

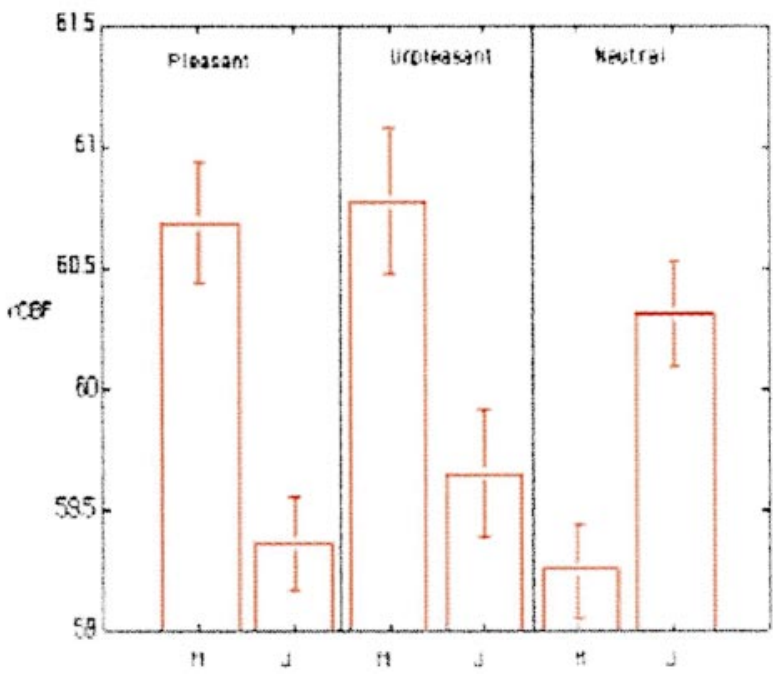

FIG. 2. The focus of significant activation in right anterior temporal cortex $(P<0.05$, corrected) is displayed superimposed upon orthogonal sections (sagittal, coronal, and transverse) of a structural scan rendered into standard space. The right lower panel provides condition specific mean corrected adjusted regional cerebral blood flow values (rCBF) for the maximum within the right anterior temporal pole for neutral and emotional conditions (displayed separately for pleasant and unpleasant) as a function of task (memory versus judgement).

This represents a three-way interaction in the factorial design where we examine the influence of target density on difference in differences between emotional and neutral conditions in the comparison of memory and judgement conditions. In this interaction the only significant effect was an activation in the left amygdala ( $\mathrm{P}<0.001$, uncorrected), extending into the uncus associated with high target emotional memory retrieval (see Fig. 3; Table 2b).

The specificity of the amygdala response to emotional item retrieval was established in a contrast restricted to the memory conditions al one invol ving a comparison of target density (high versus low) for emotional compared to neutral memory conditions. Again the only significant effect was in the left amygdala $(P<0.001$, uncorrected). This effect was independent of the valence of the retrieved material (i.e., there was no interaction in the left amygdala as a function of whether the retrieved material was pleasant or unpleasant). Finally, when testing the simple main effect of high versus low target density for emotional memory items the amygdala response remained significant $(\mathrm{P}<0.001$, uncorrected). Thus, the response profile in the amygdala indexed actual retrieval of emotional items.

Finally, to demonstrate that emotional memory retrieval activation is qualitatively distinct from retrievalrelated activations regardless of emotional valence we compared high with low target density effects for all memory conditions (i.e., memory-specific main effect of target density). This revealed a characteristic profile of bilateral prefrontal and right lateral parietal activation (all P $<0.001$, uncorrected: see Table 2c), an identical pattern to that which we have previously reported (Rugg et al., 1996). 

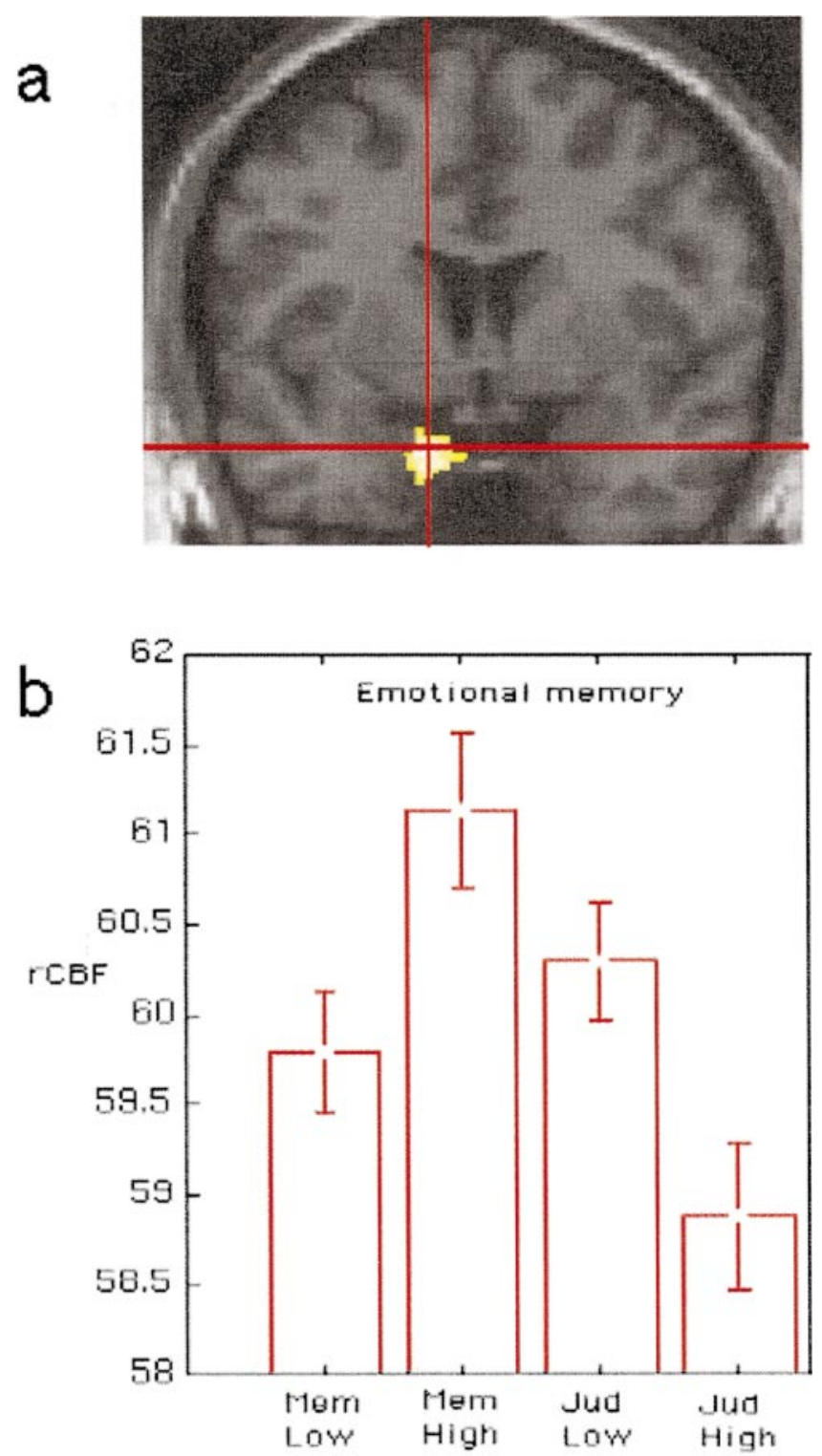

FIG. 3. The focus of significant activation in left amygdala associated with high target retrieval of emotional items $(P<0.001$, uncorrected) is displayed superimposed upon a coronal MRI image. The effect in the left amygdala is derived from an analysis of a three-way interaction involving task by valence by target density. The right lower panel provides condition-specific mean corrected adjusted regional cerebral blood flow values (rCBF) for all emotional conditions (high and low targets in memory and judgement conditions).

\section{DISCUSSION}

The prefrontal and parietal cortices and, to a lesser extent, the hippocampus have all been implicated in functional neuroimaging studies of memory retrieval (Fletcher et al., 1997). Furthermore, the profile of retrieval-related activation in these regions has been shown to be modulated by the nature of the stimulus material, for example, whether it is verbal, visual, or imageable (Fletcher et al., 1995). The current study demonstrates that emotional content of retrieved material activates neural systems qualitatively distinct from those involved in retrieving emotionally neutral material. F urthermore, this memory response profile is itself characterized by a dissociation between an emotional retrieval context response and a target density response.

The anterior temporal pole, activated to an equal extent in high and low target density memory conditions, constitutes a component of paralimbic cortex whose putative functions include imparting affective tone to experience (Mesulam, 1985). Paralimbic regions, including anterior temporal poles, receive inputs from unimodal and heteromodal sensory regions as well as so-called limbic inputs, an anatomical arrangement that could allow behavioral relevanceappraisal of sensory inputs with respect to prior memory representations (Mesulam, 1985). A more specific memoryrelated function is suggested by reports that patients with anterior temporal lobectomy manifest deficits in olfactory and autobiographical memory (Calabrese et al., 1996; Kapur et al., 1992; Rausch et al., 1977), while electrical stimulation of temporal pole induces feelings of familiarity, memory flashbacks, and autonomic responses (Chapman et al., 1950; Kaada et al., 1949; Wall and Davis, 1951; Gloor et al., 1982; Wieser, 1983). Our data indicate that the functional role of anterior temporal cortex in emotional memory retrieval relates primarily to the psychological context engendered by attempted or actual emotional item retrieval. Psychological context in this regard refers to an expectation that an emotional item will be retrieved.

Recent functional imaging data from a study of autobiographical memory has identified a right anterior temporal lobe activation similar to what we report here (Fink et al., 1996). It seems reasonable to assume that autobiographical memories automatically embody emotional qualities and this factor might account for activation of anterior temporal pole. We have also previously reported right temporal poleactivation when subjects attend to subjectiveemotional responses evoked by viewing emotional picture sets, a response that is absent when task instructions involve a spatial judgement with respect to the similar items (Lane et al., 1997). Consequently, in both these studies anterior temporal pole activation was seen in a psychological context that necessarily embodied reference to prior emotional experience.

The other region activated during emotional memory retrieval was the left amygdala. To our knowledge this is the first functional neuroimaging study that provides evidence for an amygdala role in retrieving emotional episodic memories. Our finding that amygdala retrievalrelated activation is independent of material valence complements recent evidence for a similar lack of 
differential effect at encoding (Hamann et al., 1999). A well-established view links the amygdala to emotional memory encoding. For example, there is an extensive animal literature that associates amygdala function with consolidation of emotional long-term memories (McGaugh et al., 1992; Cahill and McGaugh, 1998). In humans a role for the amygdala during memory encoding is suggested by evidence that enhanced free recall for emotional, relative to neutral, film clips is significantly correlated with an index of increased right amygdala activity at encoding (Cahill et al., 1996). Neuropsychological studies of patients with bilateral amygdala damage, indicating impaired recall for emotional material, as well as data showing preservation of the enhanced recall for emotional material in amnesics, have likewise been interpreted as supporting an encoding deficit (Babinsky et al., 1993; Markowitsch et al., 1994; Hamann et al., 1997). While these latter data are compatible with an amygdala influence at encoding it is important to bear in mind that encoding or retrieval deficits are difficult to distinguish on the basis of neuropsychological data al one. In this regard our neuroimaging data is not compatible with an exclusive role for the amygdala at encoding.

The critical question arising out of these findings is what functional role does the amygdala fulfil in emotional memory retrieval? During emotional learning the amygdala exert its effects through direct or indirect modulatory influences on cortical and subcortical structures that enhances consolidation (McGaugh et al., 1992; Ashe et al., 1989). Furthermore, this functional role is known to be time limited and shows rapid adaptation (Quirk et al., 1997; Buchel et al., 1998). Our data suggests a distinctive functional role during emotional memory retrieval. First, the relationship between target density and amygdala activation at retrieval does not easily accord with a characterization that it has a time-limited modulatory role during emotional learning. Instead, the data suggest a role during long-term retrieval, al beit following exposure to target material 5 min previously. Second, recent neuropsychological data on patients with amygdala damage highlights deficits in social judgements in response to faces, indicating that the amygdala is required for retrieval of components of social knowledge in response to visual stimuli (Adolphs et al., 1998). This social knowledge may well be evaluative in nature an idea implicit in an early conceptualization of amygdala function as attaching emotional significance to sensory stimuli (Weiskrantz, 1956). Our memory data is broadly compatible with this formulation with the added caveat that eliciting stimuli includes not only sensory events but also retrieved memories. In this light we suggest that a model for the functional role of the amygdala during memory retrieval may be to automatically index retrieved memories with representations of their past behavioral significance.

\section{ACKNOWLEDG MENTS}

R.J .D. and P.F. were supported during this work by the Wellcome Trust. Requests for materials, including I APS picture numbers, can be forwarded to r.dolan@fil.ion.ucl.ac.uk.

\section{REFERENCES}

Adolphs, R., Tranel, D., and Damasio, A. R. 1998. The human amygdala in social judgment. Nature 393:470-474.

Ashe, J . H., McKenna, T. M., and Weinberger, N. M. 1989. Cholinergic modulation of frequency receptive fields in auditory cortex: Frequency-specific effects of anticholinesterases provide evidence for a modulatory action of endogenous ACH. Synapse 4:44-54.

Babinsky, R., Calabrese, P., Durwen, H. F., Markowitsch, H. J ., Brechtelsbauer, D., Heuser, L., and Gehlen, W. 1993. The possible contribution of the amygdala to memory. Behav. Neurol . 6:167-170.

Bower, G. H. 1992. How might emotions affect learning. In The Handbook of Emotion and Memory: Research and Theory (S. A. Christianson, Ed.) Lawrence Erlbaum, Hilsdale, NJ

Bradley, M. M., Lang, P. J. 1994. Measuring emotion: The SelfAssessment Manikin (SAM) and the semantic differential. J . Behav. Ther. Exp. Psychiatry 25:49-59.

Brewer, G. H. 1988. Memory for randomly sampled autobiographical events. In Remembering Reconsidered: Ecological and Traditional Approaches to the Study of Memory (U. Neisser and E. Winograd, Eds.) Cambridge Univ. Press, New York.

Buchel, C., Morris, J ., Dolan, R. J ., Friston, K.J . 1998. Brain systems mediating aversive conditioning: An event related fMRI study. Neurology 20:947-957.

Cabeza, R., Nyberg, L. 1997. Imaging cognition: An empirical review of PET studies with normal subjects. J . Cogn. Neurosci. 9:1-26.

Cahill, L., Haier, R. J ., Fallon, J ., Alkire, M. T., Tang, C., Keator, D., Wu, J., McGaugh, J. L. 1996. Amygdala activity at encoding correlated with long-term, free recall of emotional information. Proc. Natl. Acad. Sci. USA 93:8016-8021.

Cahill, L., McGaugh, J. L. 1998. Mechanisms of emotional arousal and lasting declarative memory. Trends Neurosci. 21:294-299.

Calabrese, P., Markowitsch, H. J ., Durwen, H. F., Wilditzek, H., Haupts, M., Holinka, B., Gehlen, W. 1996. Right temporofrontal cortex as critical locus for the ecphory of old episodic memories. J . Neurol. Neurosurg. Psychiatry 61:304-310.

Chapman, W. P., Livingston, K. E., Poppen, J . L. 1950. Effect upon blood pressure of electrical stimulation of tips of temporal lobes in man. J . Neurophysiol. 13:65-71.

Fink, G. R., Markowitsch, H. J ., Reinkemeier, M., Bruckbauer, T., Kessler, J ., Heiss, W.-D. 1996. Cerebral representation of one's own past: Neural networks involved in autobiographical memory. J Neurosci. 16(13):4275-4282.

Fletcher, P., Frith, C. D., Baker, S., Shallice, T., Frackowiak, R. S. J ., Dolan, R. J . 1995. The mind's eye-Activation of the precuneus in memory related imagery. Neuroimage 2:196-200.

Fletcher, P. C., Frith, C. D., Rugg, M. D. 1997. The functional neuroanatomy of episodic memory. Trends Neurosci. 20(5):213218.

Friston, K. J ., Holmes, A. P., Worsley, K. J ., Poline, J .-P., Frith, C. D., Frackowiak, R. S. J. 1995. Statistical parametric mapping in functional imaging: A general linear approach. Hum. Brain Mapp. 2:189-210.

Gloor, P., Olivier, A., Quesney, L. F., Andermann, F., Horowitz, S. 
1982. The role of the limbic system in experiential phenomena of temporal lobe epilepsy. Ann. Neurol. 12:129-144.

Hamann, S. B., Cahill, L., Squire, L. R. 1997. Emotional perception and memory in amnesia. Neuropsychol ogy 11:104-113.

Hamann, S. B., Ely, T. D., Grafton, S. T., Kilts, C. D. 1999. Amygdala activity related to enhanced memory for pleasant and aversive stimuli. NatureNeurosci. 2:289-293.

Holmes, D. 1970. Differential change in affective intensity and the forgetting of unpleasant experiences. J . Person Soc. Psych. 15:234239.

Kaada, B. R., Pribram, K. H., Epstein, J . A. 1949. Respiratory and vascular responses in monkeys from temporal pole, insula, orbital surface and cingulate gyrus. J . Neurophysiol. 12:348-356.

Kapur, N., Ellison, D., Smith, M. P., McLellan, D. L., Burrows, E. H. 1992. Focal retrograde amnesia following bilateral temporal lobe pathology. Brain 115:73-85.

Lane, R. D., Fink, G., Chua, P., Dolan, R. J . 1997. Neural activation during selective attention to subjective emotional responses. Neuroreport 8:3969-3972.

Lang, P. J ., Bradley, M. M., Cuthbert, B. N. 1995. The International Affective Picture System (IAPS): Photographic slides. University of Florida, Gainesville, FL.

Markowitsch, H. J ., Calabrese, P., Wurker, M., Durwen, H. F., Kessler, J ., Babinsky, R., Brechtelsbauer, D., Heuser, L., Gehlen, W. 1994. The amygdala's contribution to memory-A study on two patients with Urbach-Wiethe disease. Neuroreport 5:1349-1352.

McGaugh, J . L., Introini-Collisin, I. B., Cahill, L., Kim, M., Liang, K. C. 1992. Involvement of the amygdala in neuromodulatory influences on memory storage. In The Amygdala: Neurobiological
Aspects of Emotion, Memory and Mental Dysfunction (J . P. Aggleton, Ed.), pp. 143-167. Wiley-Liss, New York.

McGaugh, J . L., Cahill, L., Roozendaal, B. 1996. Involvement of the amygdala in memory storage: interaction with other brain systems. Proc. Natl. Acad. Sci. USA 93:13508-13514.

Mesulam, M.-M. 1985. Patterns in behavioural neuroanatomy: Association areas, the limbic system, and hemispheric specialization. In Principles of Behavioral Neurology (M.-M. Mesulam, Ed.), pp. 1-70. F. A. Davis Co., Philadel phia.

Quirk, G. J ., Armony, J . L., LeDoux, J . E. 1997. Fear conditioning enhances different temporal components of tone-evoked spike trains in auditory cortex and lateral amygdala. Neurology 19:613624.

Rausch, R., Serafitinides, E. A., Crandall, P. H. 1977. Olfactory memory in patients with anterior temporal lobectomy. Cort 13:445452.

Rugg, M. D., Fletcher, P. C., Frith, C. D., Frackowiak, R. S. J ., Dolan, R. J. 1996. Differential activation of the prefrontal cortex in successful and unsuccessful memory retrieval. Brain 119:20732083.

Talairach, J ., Tournoux, P. 1988. Co-Planar Stereotaxic Atlas of the Human Brain. George Thieme Verlag, Stuttgart.

Wall, P. D., Davis, G. D. 1951. Three cerebral cortical systems affecting autonomic function. J . Neurophysi ol . 14:508-517.

Weiskrantz, L. 1956. Behavioural changes associated with ablation of the amygdaloid complex in monkeys. J . Comp. Physiol. Psychol. 49:381-391.

Wieser, H. G. 1983. Depth recorded limbic seizures and psychopathology. Neurosci. Biobehav. Rev. 7:427-440. 